\title{
Arbor
}

\section{Misiones y capacidades del Ejército del Aire en los comienzos del siglo XXI}

\section{Ricardo Rubio Villamayor}

Arbor CLXXI, 674 (Febrero 2002), 259-282 pp.

En este artículo, su autor, el General Jefe del Mando Aéreo de Combate, nos expone, desde un punto de vista esencialmente operativo refrendado por sus últimas actuaciones en conflictos y otras misiones, las características y capacidades del Poder Aéreo.

Se hacen unas reflexiones sobre las capacidades básicas que debe tener la Fuerza Aérea española para que tenga una dimensión operativa creíble, y que la hagan apta para afrontar con garantías de éxito los nuevos escenarios y retos del futuro, desde el terrorismo hasta las operaciones de paz y humanitarias.

Desmenuza las misiones que hoy desarrolla el Ejército del Aire, tanto a favor de la consecución de los objetivos de la Defensa Nacional, como en apoyo de la sociedad a la que sirve. Finalmente actualiza otros aspectos relevantes, entre los que destacan la colaboración con los Ejércitos de Tierra y Armada, la denominada "Acción Conjunta», y con los Ejércitos de otros países, "Acción Combinada», así como el cumplimiento de los compromisos militares adquiridos a nivel internacional.

\section{Introducción}

La importancia de la Fuerza Aérea por el medio que utiliza, el avión, ha ido creciendo desde su aparición como arma allá en la Primera Guerra Mundial. Su participación desde entonces ha sido cada vez más im- 
portante y decisiva a través de los conflictos en los que ha tomado parte. Últimamente, la Guerra del Golfo y el Teatro de los Balcanes han demostrado hasta que punto la Fuerza Aérea puede prácticamente por ella misma resolver un conflicto.

Hoy día cualquier doctrina militar dogmatiza que para cumplir con éxito su misión es indispensable tener la Superioridad Aérea, entendiéndose por tal la posibilidad de que las fuerzas propias de cualquier tipo puedan actuar sin ser atacadas por la Fuerza Aérea enemiga.

Alcanzar esta superioridad, es el primer objetivo de toda Fuerza Aérea. Se puede decir sin miedo a equivocarse que la falta de superioridad aérea supone la pérdida de cualquier prerrogativa en un conflicto armado. ¿ Como se alcanza esta superioridad aérea tan necesaria para el éxito propio en caso de guerra ? Por supuesto disponiendo de los medios aéreos adecuados, fundamentalmente del avión de combate que deberá tener unos requisitos que le faciliten el poder controlar el espacio aéreo al estar mejor dotado que los medios enemigos.

Para deducir cuales son estos requisitos que un avión moderno de combate (razón de ser de una Fuerza Aérea) debe tener, es necesario previamente recordar algunas de las características del Poder Aéreo para vincularlos a ellas.

\section{Características del Poder Aéreo}

Se entiende por Poder Aéreo al conjunto de medios que funcionando coordinadamente y bajo una sola dirección, se encuentra permanentemente dispuesto para destruir o neutralizar el poder y potencial militar adversario. El Poder Aéreo se materializa en la Fuerza Aérea. Veamos cuales son sus características:

\section{Velocidad}

Permite responder rápidamente a las necesidades militares completando sus misiones en muy cortos períodos de tiempo. A nivel táctico supone reducir el tiempo sobre territorio enemigo y por tanto disminuir su vulnerabilidad.

\section{Alcance/Penetración}

Basta recordar que, en la tierra el $70 \%$ está cubierta de agua, el 30\% de superficie terrestre pero el $100 \%$ está rodeado por aire, para deducir 
que el poder aéreo se puede proyectar a grandes distancias en cualquier dirección sin obstáculos de montañas o costas, allá donde los medios de superficie tendrán limitada su actuación.

\section{Flexibilidad}

El avión puede realizar muchos tipos de misiones diferentes. Ej. Un F-18 operando desde la B.A. de Valladolid puede realizar en un mismo día ataques en sitios tan diferentes como un barco a $200 \mathrm{NM}$ de la Coruña, la Isla de Alborán ( Mediterráneo), hacer un reconocimiento en Azores y realizar un combate aéreo sobre la isla de Ibiza.

\section{Elevado grado de alistamiento y respuesta}

El Poder Aéreo, puede desplegarse rápidamente a una zona de crisis para prevenir la escalada en un conflicto. Es el mejor instrumento de disuasión como manifiesto de una postura de fuerza política. En la Guerra del Golfo dos Escuadrones de F-15 se desplegaron desde EE.UU. en Arabia Saudita a las 36 horas de la invasión de Kuwait listos para entrar en combate. La ofensiva terrestre tardó seis (6) meses en poder realizarse.

\section{Destrucción}

Hoy día los medios aéreos disponen de una elevada capacidad de destrucción dada la variedad y letalidad del armamento que pueden transportar. Este armamento puede ir desde el nuclear, hasta el NBQ pasando por toda la gama del convencional dirigido o no. En este sentido, cabe destacar la gran precisión del armamento aéreo, sobre todo del inteligente, donde las precisiones en Aire/Superficie están alrededor de los 10 mts y en Aire/Aire próximo al $95 \%$ de impacto directo.

Por estas características intrínsecas, el Poder Aéreo tiene un alto valor político ya que puede gestionar rápidamente situaciones de crisis, siendo a la vez un medio decisivo y fundamental en los conflictos / guerras modernos.

\section{Capacidades operativas}

Vistas estas principales características del Poder Aéreo pasemos a considerar lo que verdaderamente constituye una de las partes principa- 
les del tema de este artículo: las Capacidades Operativas de los sistemas de armas ( aéreos) en los comienzos del siglo XXI.

En cualquier caso estas capacidades estarán siempre basadas en las citadas características positivas del poder aéreo antes mencionadas.

Para poder alcanzar la superioridad aérea, misión fundamental de la Fuerza Aérea, es necesario disponer de un avión de caza y ataque que cumpla una serie de requisitos que le permitan hacer frente a las diferentes amenazas posibles.

Estas amenazas serán:

- Misiles tipo Superficie-Aire (SAM)

- Aviones de Caza (nuevas generaciones o híbridos)

- Misiles Aire-Aire (AAM) para el combate aéreo

En cuanto a la defensa antiaérea ( AAA) de todo tipo, basta decir que de las 267 naciones del mundo, sólo 30 no disponen de este tipo de armas, las cuales pueden poner en peligro la posibilidad de alcanzar y mantener la superioridad aérea como sucedió en la guerra YOM-KI-PUR.(SAM-6)

Los aviones de combate de la última generación ( EF-2000, RAFALE, GRIPEN, SU-35, MIG's ...) son aviones que pueden ser exportados a terceros países, con relativa facilidad y poner en peligro la superioridad aérea, incluso de las grandes potencias.

Los nuevos misiles Aire/Aire (AMRAAM, MICA, A7A 12, ETC...), dadas sus características operativas « dispara y olvida «, lanzados más allá del alcance visual, incluso desde aviones híbridos (antiguos con mejöras técnicas introducidas «RETROFIT»), constituyen sistemas de aviones de bajo coste pero con un gran rendimiento operativo, pudiendo significar un grave peligro sobre todo si se emplean en número elevado.

Las principales capacidades o requerimientos específicos que tendrán que tener los sistemas de armas aéreos del futuro, en función de los grados de amenaza a los que se verán sometidos, se exponen a continuación:

\section{Tecnología STEALTH (Baja detección)}

Es conocido el éxito de los aviones que disponen de esta característica como se pudo ver con el F-117 en la Guerra del Golfo, Kosovo, donde realizaron las misiones más peligrosas y difíciles, iniciando el conflicto abierto, y destruyendo el $40 \%$ de los objetivos estratégicos con sólo un $2 \%$ de las salidas totales, de la fuerza aérea aliada.

La capacidad STEALTH, es la más importante de las características de los aviones de combate del futuro, ya que su baja detectabilidad le permite obtener la sorpresa (ver primero y disparar primero), consiguiendo degradar las fases de actividad de un radar (detección, iluminación y se- 
guimiento), tanto sean de abordo, terrestre como de los propios de misiles una vez lanzados. Asimismo, reduce drásticamente su señal I/R (infrarrojos).

En cualquier caso la tecnología STEALTH se consigue mediante:

- Bajo coeficiente de sección radar (RCS)

- Baja fuente infrarroja (I/R)

- Silueta estilizada (visual)

- Ausencia de humos y estelas

- Bajo nivel de ruido

El objetivo final es eliminar del avión todas las «firmas» posibles de detección. Se ha conseguido en aviones STEALTH (F-117, B-1, B-2 ...) tener una superficie de radar equivalente 10.000 veces más pequeña que la de un F-4 o F-15. Como ejemplo el RCS de un F-15 = $100 \mathrm{~m}^{2}$ y el de un $\mathrm{F}-117=00^{\prime} 003 \mathrm{~m}^{2}$.

Ciertos elementos de un avión se identifican como claros multiplicadores del RCS. Entre éstos caben destacar: las cargas externas y lanzadores asociados. De aquí que en los aviones con características STEALTH el armamento se transporte en bodegas internas. Los planos de deriva y profundidad (colas) por ser planos y estar colocados con ángulos grandes sobre el avión, también suponen una fuerte reflexión radar, por ello se tiende al empuje vectorial que al mismo tiempo que aumenta la maniobralidad reduce las superficies planas del avión (eliminación de colas y timones).

Como asunto curioso el personal de apoyo (mecánicos) en aviones de estas características, utiliza ropa de trabajo especial sin cremalleras ni broches, con calzado especial para no dañar el recubrimiento de la pintura especial del avión (RAM).

\section{Velocidad de crucero elevada}

El avión futuro de combate deberá poder volar a velocidad de crucero constante en supersónico (1.4/1.6 MACH) sin el empleo de los postquemadores (sobre potencia). Esto elimina señal $I / R$ y reduce el consumo de combustible, con ello se consigue:

- Disminuir el tiempo de exposición a los SAM/AAA (defensas antiaéreas)

- Acercarse al avión enemigo en el momento y lugar más adecuado.

- Recorrer más distancia en menor tiempo lo que puede significar mayor número de interceptaciones o entradas en combate, así como el abandono del mismo si la situación fuera adversa. 
Hoy día el avión de combate para mantener vuelo supersónico necesita utilizar los postquemadores con el consiguiente incremento de consumo de combustible (cuatro veces mayor que el de potencia militar), con un tiempo máximo de utilización no superior a 5 minutos.

\section{Agilidad / Maniobrabilidad}

Esta característica supone una gran ventaja a la hora de producirse un combate aéreo cerrado. Aunque el piloto debe intentar derribar al contrario principalmente mediante misiles de alcance medio (AMRAAM), esto no es siempre posible (problemas de identificación) por lo que se puede llegar al combate visual. En este caso, la agilidad y maniobrabilidad a cualquier altura será de alto valor. El avión deberá ser capaz de acelerar rápidamente, tener gran capacidad de viraje (grados por segundo sostenidos) y pequeño radio, así como soportar fuertes aceleraciones (entre + 9 G's y -3 G's), gran velocidad ascendente lo que en términos generales significa poder cambiar rápidamente la posición del avión respecto a los tres ejes de movimiento.

El «empuje vectorial» de las futuras plantas de potencia hará que los aviones sean muy maniobrables (caso del X-31) superando a los cazas de las actuales generaciones en maniobras de combate.

Asimismo, al avión se le exigirá que pueda operar desde pistas cortas, es decir con carreras de aterrizaje y despegue no superiores a los 600 mts. No será requisito imprescindible el despegue y aterrizaje verticales (VTOL), dado el grado de penalización que ello supone a la capacidad operativa del avión (elevado consumo).

\section{Versatilidad}

La tendencia actual y futura del avión es que sea MULTIROL, es decir que tenga capacidad tanto para Aire/Aire como para Aire/Suelo. No obstante, el rol primario deberá ser fundamentalmente para misiones A/A manteniéndose como secundario el de ataque al suelo. La experiencia demuestra que un avión diseñado para A/A se puede utilizar con éxito en acciones $\mathrm{A} / \mathrm{S}$, pero la inversa difícilmente se verifica.

Esta versatilidad es lógica pues una vez alcanzada la superioridad aérea el avión se empleará como arma de apoyo de la fuerzas de superficie (ET y/o Armada), e incluso puede haber situaciones tácticas donde las operaciones A/S tengan prioridad. 
En el futuro las características de un avión de caza (superioridad) en cuanto a su agilidad no se penalizarán como hoy día, dado que las armas tanto A/A como A/S irán de forma interna en el avión.

\section{Radio de acción}

Esta característica tiene mucha importancia ya que un avión con gran radio de acción puede operar desde bases alejadas del territorio enemigo (reduciendo su vulnerabilidad) e incluso alcanzar desde un menor número de Bases Aéreas propias, objetivos situados en uno e incluso varios teatros de operaciones, lo que además simplifica el problema de apoyo logístico.

El empleo de aviones cisternas, cada vez más extendido, permite obviamente mejorar el radio de acción de los aviones o permanencia en el aire (CAP). En cualquier caso el radio de combate que se exigirá a los aviones de caza no será inferior a $600 \mathrm{NM}$ (0 $1000 \mathrm{Kmts})$ en configuración tanto Aire/Aire como Aire/Suelo.

\section{Aviónica moderna y avanzada}

Se entiende por aviónica, en un sistema de armas aéreo, los equipos correspondientes a:

- Comunicaciones

- Identificación

- Navegación

- Sistemas de Armamento (Radar/Tiro)

- Guerra Electrónica

En el futuro a los aviones se les exigirá que:

- Las comunicaciones sean seguras (secrafonía) y fiables (redundancia).

- Los sistemas de identificación propios deberán ser también altamente fiables mediante equipos electrónicos (IFF/SIF), I/R y ECM.

- La navegación tendrá que ser completamente autónoma basada principalmente en sistemas integrados GPS-INS.

- Los sistemas de armamento y tiro altamente precisos, incluso con armamento convencional. En este caso los errores deberán ser inferiores a los 10 metros en caída libre, mediante el cálculo constante de la trayectoria y punto de impacto.

- Dispongan de un radar multimodo capaz de seguir simultáneamente en $\mathrm{A} / \mathrm{A}$, un número de blancos similar al de misiles disponi- 
bles, con capacidad LOOKDOWN y alcance no inferior a los 100 kms. Este radar debe ser complementado con un sensor I/R para búsqueda y seguimiento.

- En el área de guerra electrónica, deberá estar dotado de medios activos (perturbadores) y pasivos (o de detección) así como de engaño (lanzadores de CHAFF y bengalas o remolque de sueñuelos). Todos estos medios deberán ir incorporados dentro de la estructura del avión.

\section{Propulsión}

La gran autonomía, añadida al requisito paralelo de mantener altas velocidades de crucero, hace que la elección del motor sea uno de los puntos más críticos de un sistema de armas.

Los futuros aviones de combate incorporarán toberas para control de empuje variable (maniobrabilidad); excelente aceleración para cambios rápidos de velocidad; comportamiento seguro para grandes ángulos de ataque; deberán soportar fuertes factores de carga y aceleración, así como maniobras de desplazamiento lateral libres de perdidas de compresor. No deberá dar señal de humos, ni de utilización de los postquemadores. Debe poder operar hasta 10 horas continuas (con reabastecimiento en el aire), sin límites de utilización.

¿Un motor o dos?

Este es un aspecto importante a la hora de tener en cuenta la seguridad y supervivencia del avión. No obstante para su consideración habrá que analizar dos factores: precio y fiabilidad (posibilidad de fallo). El segundo motor supondrá siempre un incremento notable de precio y complicaciones técnicas añadidas. Por otro lado habrá que tener en cuenta que los componentes del motor son cada vez más fiables (MTBF), lo que garantiza la operatividad del avión con uno solo. En este sentido, hay que considerar que hoy día se exige a los motores una fiabilidad tal que la tasa de fallos debe de ser menor a 1 fallo por cada 500 horas de funcionamiento y éste no debe implicar cambio de motor. El cambio de un motor se deberá hacer en un tiempo inferior a 20 minutos.

\section{Supervivencia}

Se puede lograr reduciendo, como ya se ha explicado, su observabilidad/detección (STEALTH). Para ello se deberá cuidar: 
- La configuración externa, ausente de puntos para cargas exteriores, antenas, etc.

- Diseño adecuado del motor con especial cuidado de las tomas de aire y salida de gases, sin perjudicar el rendimiento del mismo.

- Dotar de un pequeño radar que cubra el sector de cola.

- El control de las emisiones en los sistemas de comunicaciones, navegación e identificación.

- Utilización de bodegas conformadas para transportar el armamento, etc.

\section{Armamento}

Conceptualmente al avión del futuro se le pedirá que utilice generalmente sólo armamento inteligente, tanto para operaciones A/A como para A/S. Se ha abandonado la idea de alcanzar un blanco por saturación por la de «un objetivo, un arma», dada la alta precisión del armamento actual, guiado por diversos medios (TV, I/R, LASER, GPS, INS, etc.) lo que además de reducir el esfuerzo aéreo permite evitar los daños colaterales, factor este muy importante en las cada día más frecuentes Operaciones de Paz. Estas armas deben poder ser lanzadas a una distancia del objetivo que permita evitar sus defensas (STAND-OFF), debiendo ser las precisiones inferiores a los $10 \mathrm{mts}$ para acciones A-S.

Las armas deberán ser específicas para la destrucción de objetivos de diferentes características (rompedoras, antitanque, antibuque, antipistas, antipersonal, antirradar, de penetración, incendiarias, negación de área etc.) todas ellas guiadas y lanzadas a distancia.

Dada la precisión de estas armas, al avión no se le exigirá que transporte más allá de 2.000 kgs de armamento por salida.

\section{Cabina de vuelo (Pilotaje)}

El piloto que se siente en la cabina de un futuro avión de combate tendrá en todo momento sus manos ocupadas. Estas descansarán sobre empuñaduras de cuidado diseño ortopédico erizadas de botones que permitirán controlar en todo momento las evoluciones del avión, el control de motor y de todos los sistemas: identificación ,armamento, autoprotección, etc.

La cabina dispondrá de varias pantallas de cristal líquido, multicolores (LCD) así como de un visor transparente (HUD), proyectando sobre el parabrisas y de múltiples señales acústicas y avisadores luminosoś 
que proporcionarán al piloto la información necesaria que le permita cumplimentar su misión con garantía de éxito y seguridad.

Estas pantallas de cristal líquido (LCD), son más ligeras, pequeñas y permiten un $40 \%$ más de información con menos gasto, que las típicas de rayos catódicos (tubo), debiendo representar situaciones de navegación, estado de armamento y amenazas, sistemas del avión radar /IR etc. Cabe destacar que el índice de averías de estas pantallas es muy bajo (alrededor de 5.000 horas MTBF).

La cúpula/parabrisas del avión será protegida contra el choque de pájaros $(1 \mathrm{~kg}$ a $800 \mathrm{k} / \mathrm{h}$ ). Además, la cabina del piloto llevará protección NBQ.

\section{Equipo personal}

Las altas características de vuelo de los modernos aviones hacen necesario proteger al piloto en el entorno ambiental en el que va a estar sometido. En este sentido debe protegerse principalmente ante los efectos de las fuertes aceleraciones (cargas G's), para ello el piloto vestirá un traje anti $\mathrm{G}$ integral y dispondrá de un sistema de respiración forzada automático.

Asimismo el piloto dispondrá de un casco de vuelo, donde en la visera y en forma transparente, se proyectarán los datos tanto de vuelo como aquellos otros de armamento y disparo que seleccione previamente, lo que le permitirá realizar los combates sin tener que maniobrar el avión para apuntar al enemigo, e incluso observar todo su entorno (de combate) controlando simultáneamente los parámetros de vuelo y de puntería sin obligar a forcejeadas maniobras de avión.

Un sistema que se impondrá en el futuro es el denominado «VOICECOMMAND», es decir un equipo que permitirá al piloto seleccionar ciertos modos de los equipos de abordo, fundamentalmente los referidos a comunicaciones, navegación e identificación a órdenes de la propia voz, lo que le descargará de «trabajo manual» (HOTAS).

Dispondrá de un asiento eyectable tipo «cero-cero» (altura cero/velocidad cero), con estabilizadores de posición apertura automática y dotado de equipo de supervivencia. Deberá ir lo más reclinado posible (confort y tolerancia a G's).

El piloto no requerirá ayuda del mecánico para instalarse en la cabina.

\section{Mantenimiento y disponibilidad}

En términos generales, el ciclo de vida de un futuro avión se cifrará en 25 años y 6.000 horas de vuelo. 
Uno de los requisitos más exigentes para estos aviones será el tener un índice de mantenimiento bajo, es decir que se necesiten muy pocas horas de trabajo en relación con el número de horas de vuelo. Como ejemplo podemos decir que así como para un avión de los años 60 (RF4C) se requería 40 horas / hombre por hora de vuelo; un F-18, hoy día requiere 20 horas / hombre; pero un EF-2000 requerirá no más de 9 horas/hombre.

En términos generales a los aviones del futuro se les exigirá que puedan realizar mayor número de salidas con menos especialistas y equipos de mantenimiento que los de hoy día, para ello tendrá un elevado grado de autonomía disponiendo de equipos incorporados al propio avión (APU, Oxígeno, Bancos de Prueba,...).

Los diferentes componentes que conformen el sistema de armas tendrán un menor índice de fallos, llegándose a fiabilidades (MTBF) de 6.000 horas de vuelo (es decir casi iguales a las del propio ciclo de vida del avión). Ejemplo de esta alta fiabilidad es el equipo GPS-INS del F117 que tiene un MTBF comprobado de 2.000 horas.

Todo ello significa que los despliegues se podrán hacer de forma más independiente, es decir con menos medios, tanto humanos como materiales (apoyo logístico).

Los tiempos de recuperación para volver al combate, es decir el reabastecimiento de combustible y armamento del avión, así como su comprobación, serán inferior a 20 minutos con seis (6) hombres para acciones A/A y 30 minutos para A/S.

En este aspecto, conviene hacer constar que si bien en principio el costo de los sistemas de armas aéreos se puede considerar elevado, no lo es tanto si se tiene en cuenta que el ciclo de vida es muy largo. Como ejemplo cabe mencionar los aviones B-52 y B-707 que estarán operativos por más de 50 años, o nuestros aviones F-5, F-1 o C-130 estarán asimismo operativos más de 40 años. En cualquier caso se puede decir que la relación coste / eficacia es altamente beneficiosa.

\section{Capacidades básicas de una Fuerza Aérea}

El Ejército del Aire, para que tenga una dimensión operativa creỉble, además de las mencionadas capacidades de los aviones de combate, deberá contemplar también los siguientes aspectos que se consideran básicos:

- Medios para proporcionar «Alerta Temprana», fundamentalmente aerotransportada, para potenciar la Defensa Aérea (Sistema de Vigilancia). 
- Armamento moderno, para optimizar el esfuerzo aéreo (menor número de salidas y aviones), así como evitar/reducir los «daños colaterales».

- Entrenamiento de las tripulaciones y personal de apoyo, lo más realista posible.

- Adecuada capacidad de transporte y reabastecimiento aéreo para potenciar tanto la flexibilidad como el alcance de los aviones de combate y reconocimiento.

- Un sistema de Mando y Control e Inteligencia que cubra todas las necesidades del concepto «CIS». Este sistema deberá ser interoperable con el de nuestro aliados.

- Un sistema logístico capaz, que pueda apoyar operaciones continuadas y realizadas lejos de las bases permanentes, y en cualquier condición climática.

- Por último y lógicamente, disponer de una fuerza de aviones de caza y ataque moderna y completa.

Todo lo anteriormente expuesto tiene que estar a su vez complementado por:

- Una «Doctrina Aérea» que sirva como guía para el empleo pertinente de la fuerza tanto en paz como en crisis o guerra. La doctrina no es sino una acumulación de conocimientos y experiencias logradas en análisis de las propias actuaciones en planes de instrucción, ejercicios e incluso en acciones reales de crisis o conflictos.

- Personal suficientemente motivado con una gran moral y adiestramiento adecuado.

Otras consideraciones importantes para el empleo satisfactorio de la Fuerza Aérea son:

- Las reglas de enfrentamiento (ROE) proporcionan el marco de referencia para el empleo controlado del poder militar y son el nexo de unión entre los objetivos políticos y la aplicación de la fuerza. De ahí que tienen que ser cuidadosamente redactadas, con claridad y precisión, para que sean inequívocamente comprendidas, difundidas y estrictamente aplicadas.

- El empleo del poder aéreo requiere una infraestructura operativa como requisito casi imprescindible para operar, debiendo incluir medios desplegables.

- Es necesario remarcar la importancia que para el éxito de las operaciones aéreas tiene la Estructura de Mando. En este sentido, la 
actual estructura operativa de las Fuerzas Armadas españolas supuso, con su implantación, un paso importante para lograr la máxima eficacia del instrumento militar, al separar las funciones administrativas y logísticas de las de la Fuerza, en su empleo operativo.

\section{Escenarios}

El primer y principal escenario de actuación, aunque hoy más remoto, es el derivado de defender la soberanía, independencia e integridad nacional, así como sus intereses vitales allá donde fuera necesario.

Un segundo escenario es el determinado por las operaciones de Mantenimiento de $\mathrm{Paz}$ o de carácter humanitario, auspiciados por las Naciones Unidas.

El tercer escenario es consecuente con el sistema de Seguridad Europea, ligada a las organizaciones internacionales como la OTAN, UEO.

\section{Nuevos retos}

Como ya se ha expuesto, en las circunstancias actuales, los riesgos o amenazas a un Estado pueden incluir un amplio espectro de acciones hostiles que pueden ir desde violaciones de tratados hasta agresiones armadas, incluyendo el empleo de armamento de destrucción masiva, o acciones terroristas a gran escala como la sucedida en Nueva York el pasado 11 de septiembre de 2001.

Del análisis de la situación política internacional y considerando factores de carácter geoestratégico, económico, étnico, religioso, etc., se pueden deducir las siguientes posibles nuevas situaciones en las que las FAS se podrían ver envueltas. (CUADRO 1)

Por otra parte, la situación actual de Rusia y de sus antiguas Repúblicas (Unión Soviética) facilita que tecnología avanzada militar esté disponible en países inestables o conflictivos, lo que puede convertir los focos de riesgos, sobre todo los del Tercer Mundo, en altamente peligrosos. No olvidemos que para conseguir un misil inteligente con carga de destrucción masiva, basta incorporar a un viejo «SCUD» un receptor GPS comercializado y algún elemento más poco costoso, para alcanzar con precisión un objetivo. De ahí que las tensiones, crisis y conflictos en estas áreas demanden de los países del primer mundo la participación en las llamadas «Operaciones de Mantenimiento de Paz». 


\section{CUADRo 1}

\section{Conflictos asimétricos o de baja intensidad:}

Violación de los tratados sobre control armamentos.

Agresiones mediante acciones de misiles o aviones de carácter limitado.

Ataques contra barcos y acciones en espacios aeromarítimos de carácter internacional.

Violaciones de agua o espacios aéreos de soberanía nacional.

Actos de terrorismo o sabotaje.

Amenazas o acciones hostiles contra las líneas de comunicación internacionales o aliadas.

Participación en Operaciones de Paz o Ayuda Humanitaria.

Conflictos simétricos o de alta intensidad:

Guerras de carácter convencional o incluso nuclear.

\section{Características de los futuros conflictos}

En base a lo anteriormente expuesto vemos que los conflictos a los que España como miembro de la OTAN, UE o en apoyo de resoluciones de la ONU, tendrá que hacer frente con mayor probabilidad en los años venideros, serán los denominados de baja intensidad o subconvencionales, entendiendo por éstos los que presentan las siguientes características:

- Nacen dentro de un Estado.

- Producen violaciones de las leyes internacionales, así como de los derechos humanos.

- No existe un claro agresor.

- No suelen combatir fuerzas regulares, ni bajo una sola autoridad.

- Las acciones son de tipo guerrilla o terrorismo.

- Tienen difícil solución racional y las sanciones económicas o embargos son poco eficaces.

- Son originados generalmente por fundamentalismos o nacionalismos radicales, y tienen larga duración.

\section{Terrorismo: nueva amenaza}

Los atentados terroristas del 11 de septiembre en EE.UU. están obligando a los países a tomar medidas contra esta nueva amenaza. En este sentido se aceleran los estudios y análisis para adaptar este riesgo potencial en el Concepto Estratégico de alianzas y naciones como un nuevo escenario, examinando cómo remediar las carencias en las capacidades 
Misiones y capacidades del Ejército del Aire ...

civiles y militares para definir una nueva política de Defensa y Seguridad.

Muy probablemente la lucha terrorista será desde ahora uno de los principales objetivos de la Política de Defensa y Seguridad de los Estados. En esta política habrá que conjugar tres (3) aspectos generales: Legalidad, Economía y Fuerza. Es en este último punto en el que las FAS pueden ser empleadas en acciones concretas contra el terrorismo.

Los atentados terroristas citados han hecho que se desdibujen los sistemas de defensa y seguridad tradicionales. Antes, los países se defendían de los riesgos internos con las fuerzas de Seguridad del Estado, mientras que de la defensa contra enemigos exteriores se responsabilizaba a las FAS. La lucha contra este tipo de riesgos será larga, y debe ser terminada; no se puede dejar a medias, pues sería un triunfo para los terroristas.

A partir de ahora, habrá que integrar ambas fuerzas, las de Seguridad del Estado y las de los Ejércitos.

¿Cómo se podrían ver involucradas las FAS en la lucha contra el terrorismo, en acciones similares a las sucedidas el 11 de septiembre en USA?. En este sentido, hay que considerar tres tipos de posibles acciones contra este tipo de amenaza:

- Acciones preventivas, basadas fundamentalmente en medidas policiales y en la obtención y explotación de la Información/Inteligencia sobre los grupos terroristas. Corresponden principalmente a las Fuerzas de Seguridad del Estado.

- Acciones indirectas, de tipo coercitivo o de presión, con el fin de erradicar por otros medios el terrorismo. Entre ellas, cabe destacar las de tipo político, diplomático y económico.

- Acciones directas, en las que las FAS buscarían alcanzar los objetivos concretos de estas facciones terroristas, como está sucediendo en los bombardeos de Afganistán.

En los casos de los secuestros aéreos y la utilización de aviones como armas de destrucción masiva, la Fuerza Aérea puede jugar un papel importante aunque difícil. En este sentido los aviones que realizan misiones de "policía aérea» pueden ser empleados para identificar los citados aviones, e incluso tomar las acciones pertinentes si su conducta se llegara a considerar como «acto hostil». Asimismo, se pueden tomar otras medidas preventivas, como declarar, «Zonas de Exclusión de Vuelos» a aquellas que tuvieran un alto valor estratégico, político, económico o cuya destrucción pudiera producir elevados daños colaterales. Estas «Zonas de Exclusión» podrían ser defendidas, adicionalmente, con defensas aéreas puntuales (AAA/SAM). 
En estas circunstancias se plantea la dificultad de cual es la autoridad que tiene que declarar la orden de acometimiento y disparo, teniendo en cuenta, que dada la velocidad de los medios aéreos, el tiempo para reaccionar será muy corto.

En cualquier circunstancia, en los secuestros aéreos, deberá existir una fuerte cooperación y coordinación entre controladores aéreos civiles y militares, ya que serán aquellos los que perciban en primer lugar este tipo de hechos. En el caso de nuestro Ejercito del Aire, esta cooperación existe de hecho al trabajar juntos físicamente los citados controladores.

\section{Operaciones de Paz / Humanitarias}

Las operaciones militares auspiciadas por organismos internacionales en este tipo de conflictos, se han pasado a denominar Operaciones de Paz o Humanitarias, y pueden tener diversas formas, que a continuación se exponen.

\section{Prevención de conflictos}

Requiere fundamentalmente diplomacia para evitar la escalada a un conflicto mayor. La misión militar será de apoyo a las acciones diplomáticas, pero no asume un papel predominante. En este sentido, podrán estar incluidas algunas de las capacidades militares tales como: observadores, vigilancia (satélite y radar), reconocimiento, etc., o bien la entrega y distribución de ayuda y recursos para la asistencia a la población civil.

\section{Mantenimiento de la Paz}

Se deriva, normalmente del estado anterior (prevención de conflictos). En estas operaciones la presencia militar es crítica para evitar el estallido de la violencia. Estas fuerzas militares deberán estar dotadas de gran flexibilidad y movilidad operativa, que les permitan una intervención rápida y controlar a tiempo a las fuerzas en litigio. Para ello deberán estar también dotadas de medios de vigilancia y reconocimiento que les faciliten la información precisa para actuar a tiempo.

\section{Imposición de la Paz}

Estas Operaciones se utilizan para restablecer la paz en un área de conflicto mediante el uso de las FAS, pudiéndose desarrollar tanto dentro del territorio en conflicto como fuera de él, mediante embargos y bloqueos. 
En términos generales, el uso de la fuerza militar en este tipo de conflictos, puede alcanzar prácticamente todos las formas: fuego, reconocimiento y transporte. El método y grado de aplicación pueden estar fuertemente marcados por condicionantes políticos.

\section{Construcción de la Paz}

Es un proceso marcadamente político que trata de crear un sentimiento de paz y confianza entre partes en litigio. La misión de las FAS, en este caso, es asegurar que las partes cumplan los acuerdos previamente aceptados. En este sentido, las acciones militares se limitarán a vigilancia y reconocimiento; transporte de refugiados y enseres, así como a la participación de especialistas que ayuden a la reconstrucción del país, etc.

\section{Operaciones Humanitarias}

El objetivo principal de este tipo de operaciones es, en primer lugar, aliviar las necesidades humanas primarias tales como comida, albergue o asistencia médica básica. Así mismo, las acciones militares se orientan a crear unas condiciones más estables y así prevenir un posible conflicto. En este caso, las operaciones estarán caracterizadas por las ayudas y suministros en áreas remotas, empleando la fuerza, si fuera necesario, para proteger o crear un entorno favorable que las facilitara.

\section{Ventajas de la F.A. ante los nuevos conflictos}

Las características ya mencionadas del Poder Aéreo permiten que la Fuerza Aérea pueda maniobrar en la tercera dimensión para concentrar el esfuerzo en el tiempo y en el espacio, allá donde sea necesario. Para la fuerza aérea no hay fronteras naturales, ni líneas de costa; todo está a su alcance y no hay operación de superficie que pueda desarrollarse sin alcanzar primero el grado de dominio del aire necesario. La velocidad junto con la movilidad y el alcance son características esenciales para resolver una crisis, por cuanto significa la presencia de fuerzas de interposición en el momento preciso.

Por otra parte, la capacidad de penetración en cualquier espacio hostil y la precisión y letalidad de los sistemas de armas actuales, permiten aplicar su potencia de fuego, normalmente sin causar daños colaterales o produciendo los mínimos. 
Además, la fuerza aérea puede actuar de una forma muy visible, tanto de una forma puramente defensiva, no provocativa, o disuasoria, como estamos viendo actualmente en la ex-Yugoslavia con la operación «ALLIED FORCE» (para misiones de defensa aérea (NO FLY ZONE)) y de apoyo (CAS).

Por último, la fuerza aérea puede actuar desde fuera del territorio en conflicto sin exponer fuerzas sobre el terreno y facilitando así la desescalada o retirada del conflicto. No hay posibilidad de convertirse de pacificadores en rehenes.

\section{Posibilidades del empleo de la Fuerza Aérea}

En este sentido, las misiones que han de ser realizadas en cada tipo de operación dependen de las características específicas de éstas. Por ejemplo, las operaciones de imposición de la paz son las únicas en las que se emplearía todo el espectro de una fuerza aérea y no siempre sin limitaciones. Las de diplomacia preventiva, mantenimiento de la paz, construcción de la paz y humanitarias, requieren una aplicación muy selectiva; normalmente serán más de apoyo a las operaciones (transporte aéreo, $\mathrm{SAR}$, vigilancia y reconocimiento) que de acciones que lleven consigo el empleo de armamento.

De una manera esquemática y solo con el fin de dar una idea general de las posibilidades éstas se recogen en el cuadro 2.

Obsérvese que las operaciones de reconocimiento y vigilancia, y apoyo a las operaciones siempre son necesarias.

Como puede apreciarse en lo anteriormente expuesto, la Fuerza Aérea puede emplearse en toda clase de conflictos, sean del grado que sean, e incluso para solventar y gestionar situaciones previas a los conflictos (CRISIS). Asimismo, la Fuerza Aérea puede utilizarse de forma conjunta con los ejércitos de superficie o de forma independiente. Por ello, se puede decir que el Poder Aéreo, por sus características, es un instrumento militar que puede influir positivamente en situaciones determinadas en cualquier momento y lugar que fuera necesario y lo puede hacer sin necesidad de una presencia permanente sobre el terreno en litigio.

En cualquier caso, como siempre ha de ocurrir en el empleo del poder militar la aplicación de la fuerza aérea ha de hacerse con un ajuste muy preciso a los objetivos políticos que se persiguen, con estricta sujeción a las limitaciones que se establezcan, y contemplando todas las alternativas de evolución de la crisis para ser capaces de escalar y desescalar según convenga. En este último sentido, volvemos a insistir en la 


\section{CUADRo 2}

\begin{tabular}{|c|c|c|c|c|c|c|c|}
\hline & & \multicolumn{6}{|c|}{ OPERACIONES AÉREAS } \\
\hline & & $\mathrm{DCA}$ & OCA & RAS & SAO & INT & CAS \\
\hline \multirow{3}{*}{$\begin{array}{l}0 \\
\mathrm{P} \\
\mathrm{E} \\
\mathrm{R} \\
\mathrm{A} \\
\mathrm{C} \\
\mathrm{I} \\
\mathrm{O} \\
\mathrm{N} \\
\mathrm{E} \\
\mathrm{S}\end{array}$} & $\begin{array}{l}\text { PREVENCIÓN DE } \\
\text { CONFLICTOS }\end{array}$ & & & * & * & & \\
\hline & $\begin{array}{l}\text { ESTABLECIMIENTO } \\
\text { DE LA PAZ }\end{array}$ & & & * & * & & \\
\hline & $\begin{array}{l}\text { MANTENIMIENTO } \\
\text { DE LA PAZ }\end{array}$ & * & & * & * & & * \\
\hline \multirow{2}{*}{$\begin{array}{l}\mathrm{P} \\
\mathrm{A} \\
\mathrm{Z}\end{array}$} & $\begin{array}{l}\text { IMPOSICIÓN } \\
\text { DE LA PAZ }\end{array}$ & * & * & 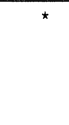 & * & $\bar{*}$ & * \\
\hline & $\begin{array}{l}\text { CONSTRUCCIÓN } \\
\text { DE LA PAZ }\end{array}$ & & & * & * & & \\
\hline
\end{tabular}

flexibilidad de empleo del Poder Aéreo ya que la amplia gama de operaciones que puede desarrollar permite proyectar el potencial militar en cualquier momento con gran rapidez de respuesta y gran precisión, incluso a grande distancias, de forma que pueda influir decisivamente desde el aire y espacio , sobre una situación determinada, en el tiempo y lugar preciso para la defensa de los intereses nacionales. Por todo ello se puede considerar al Poder Aéreo como una extraordinaria «herramienta política».

\section{Misiones específicas del E.A.}

Teniendo en cuenta las características principales y capacidades descritas anteriormente, el Poder Aéreo puede llevar a cabo los siguientes roles y misiones dentro del amplio espectro de las operaciones aéreas. 


\section{Policía del aire}

La policía del aire es una misión permanente. Es llevada a cabo tanto en paz como en situaciones de crisis. Consiste principalmente en la detección, identificación, interceptación o control de aviones no identificados que entran en el espacio de responsabilidad. El interceptador podría recibir la orden de forzar al avión interceptado a abandonar dicho espacio aéreo o a tomar tierra para una inspección. Otra faceta de la policía del aire es asistir o rescatar aviones militares o civiles en situaciones de emergencia.

\section{Defensa Aérea (DCA)}

Las misiones DCA son misiones reactivas llevadas a cabo por cazas de interceptación u otros medios (por ejemplo defensas aéreas basadas en superficie) en el aire o en alerta en tierra, para ser dirigidos contra plataformas hostiles cuando éstas sean detectadas.

\section{Operaciones Ofensivas contra la Fuerza Aérea (OCA)}

Las misiones OCA consisten en el uso de las fuerzas aeroespaciales en contra de misiles o plataformas aéreas hostiles, incluyendo el ataque a sus infraestructuras de apoyo, (bases aéreas o instalaciones de mando y control).

\section{Supresión de Defensas Aéreas (SEAD)}

Las misiones SEAD consisten en la neutralización, destrucción o degradación del sistema de defensa superficie-aire del enemigo. Las operaciones SEAD permiten que otras operaciones aéreas se realicen sin pérdidas innecesarias.

\section{Interdicción Aérea (AI)}

Las misiones AI son una modalidad de acciones aéreas que destruyen, interrumpen, desvían o retrasan el potencial militar del enemigo en superficie antes de que pueda ser empleado efectivamente en contra de las fuerzas propias.

\section{Apoyo Aéreo Cercano (CAS)}

La misión CAS es el uso de los medios aéreos para el apoyo directo a las fuerzas terrestres. Se lleva a cabo contra objetivos que se encuentran en las proximidades de las fuerzas propias, por lo cual requiere una precisa coordinación. Estas misiones deberán ser utilizadas únicamente 
cuando las fuerzas terrestres no sean capaces de hacer frente al enemigo con su potencia de fuego orgánica.

Las misiones CAS que fueron voladas en Kosovo no siguieron la definición anterior debido a que no existían fuerzas terrestres propias en el área del conflicto. En Kosovo el Poder Aéreo en solitario fue utilizado para encontrar, identificar y destruir fuerzas desplegadas, misión que de otra forma hubiera sido ejecutada por fuerzas terrestres y el CAS volado en apoyo de dichas fuerzas.

\section{Reconocimiento y vigilancia}

Las plataformas aéreas y espaciales pueden vigilar diferentes teatros de operaciones de manera visual o electrónica (encubierta o no), desde dentro o fuera del teatro.

El reconocimiento y vigilancia con plataformas aéreas tripuladas o no tripuladas y satélites, tácticos o estratégicos, operando con diferentes sensores (ópticos, infrarrojos, radar) proporcionan información esencial y en tiempo adecuado para la toma de decisiones políticas y militares.

\section{Guerra Electrónica (EW)}

Las operaciones EW implican el empleo militar de medios electrónicos para determinar, explotar, reducir o prevenir el uso hostil del espectro electromagnético y asegurar el uso efectivo de este espectro en beneficio propio.

Los tres principales roles de EW son: medidas electrónicas de apoyo ESM, contra medidas electrónicas, ECM y medidas de protección por parte de las fuerzas propias, EPM.

Combinadas con el alcance y altitud, las operaciones EW aéreas pueden cubrir la totalidad del teatro de la operación.

\section{Transporte (TCC)}

Las operaciones de transporte incluyen los movimientos aéreos de personal y carga desde, hacia, dentro o entre teatros de operaciones.

Estas misiones no sólo son exclusivamente el medio de transporte de tropas de combate y su apoyo logístico sino que pueden ser utilizadas para el transporte de alimentos y ayudas de emergencia, para realizar operaciones aeromédicas, aerotransportadas o especiales o para evacuar personal en peligro. 
El transporte aéreo puede ser estratégico o táctico, usando aviones de ala fija o helicópteros.

\section{Reabastecimiento Aire-Aire (AAR)}

Las misiones AAR permiten aumentar el alcance, autonomía, tiempo de misión, la flexibilidad de los aviones y la capacidad para redesplegar.

Estas misiones son verdaderas multiplicadoras de fuerza.

\section{Rescate Aéreo de Combate (C-SAR)}

Las operaciones CSAR suponen la utilización de aviones para rescatar a una tripulación en territorio hostil, que haya tenido que abandonar su avión.

Debido a la complejidad de esta misión, y siendo una operación llevada a cabo en territorio hostil, estas operaciones normalmente incluyen Fuerzas Especiales, con equipos de combate y de rescate adecuados. También pueden incluir el empleo de fuerzas de combate como helicópteros de ataque, cazabombarderos y medios SEAD para dotar de cobertura de fuego a las operaciones para rescatar el avión. Este tipo de misiones es un gran incentivo moral para las tripulaciones que tienen que volar sobre territorio enemigo.

\section{Otros aspectos relevantes de las FAS}

Nuestra libertad y seguridad Nacional se encuentran estrechamente unidas a la de nuestros socios aliados. Por ello, habrá que asumir mayores niveles de responsabilidad fuera de nuestras fronteras como factor determinante de nuestra presencia internacional. En este sentido se tendrá que armonizar nuestras capacidades militares con la de nuestros aliados, así como racionalizar las estructuras de defensa e incrementar la «Acción Conjunta». Asimismo, se deberá dotar a la Fuerza Aérea de medios modernos y suficientes. Esta modernización significa interoperabilidad, es decir, que los sistemas sean compatibles no solo con los de otros Ejércitos (E.T. y ARM), sino también con los de nuestros aliados; Flexibilidad, para que el ejército pueda ser empleado en diversos escenarios y operaciones; Proyectabilidad, o estar permanentemente preparados para eventuales misiones; Información, para lo que se debe tener conocimiento lo más exhaustivo posible de las condiciones en las que la fuerza se va emplear. Por ultimo, modernización es tecnología, funda- 
mental en operaciones modernas, donde cada vez es mayor la exigencia de reducir al mínimo, tanto bajas como daños colaterales.

Por otra parte, la constante disminución de los presupuestos de defensa, lleva consigo un proceso generalizado de reducción de efectivos militares. En estas condiciones es necesario la búsqueda de procedimientos que permitan obtener mayores rendimientos de los escasos recursos militares. En este sentido, la idea de lo conjunto, normalmente ligada a necesidades de carácter operativo, se plantea hoy día como una meta económica que puede ser causa de cambios notables en la organización de las FAS.

Este concepto de "Acción Conjunta», orientada a potenciar la interoperabilidad, capacidad de proyección, información, velocidad, y elementos de fuego de gran precisión, requiere una nueva doctrina que haga todo ello posible. Esta «Doctrina Conjunta» requiere un conocimiento y seguimiento de los factores de fuerza y debilidad de cada componente para obtener la máxima capacidad del esfuerzo general.

\section{Conclusiones}

El fin de la bipolarización, ha hecho menos probable el riesgo de enfrentamiento convencional o nuclear de carácter generalizado. No obstante, durante el periodo de transición que vivimos hacia otro orden internacional, nos ha llevado a un mundo inestable, conflictivo y lleno de riesgos para la seguridad.

Por otro lado, el mundo de nuestro entorno, concretamente el Europeo, se dirige hacia una globalización a todos los niveles, suprimiendo fronteras, generalizando mercados, creando la moneda única etc. También la seguridad y defensa, van dejando de ser un asunto nacional para ser cada vez un más un aspecto colectivo. Aparecen nuevas amenazas, o focos de riesgo, en forma de conflictos étnicos o religiosos, terrorismo, caos...

En todo caso la diferencia en la utilización de los Ejércitos, en los diferentes niveles de conflicto, radica únicamente en la intensidad del esfuerzo y en las Reglas de Enfrentamiento (ROE's), es decir en la forma de empleo.

Nuestro Ejercito del Aire ha dejado esto bien patente en su participación en el Teatro de los Balcanes, donde ha llevado a cabo toda clase de misiones (fuego, reconocimiento y transporte), en fases tanto de alta como de baja intensidad.

En el futuro, esta claro que la participación será integral de los tres ejércitos , (Tierra, Mar y Aire), y no sólo a escala nacional sino que tendrá 
carácter multinacional. Es decir, las operaciones militares serán conjunto/combinadas, lo que nos conduce a que el empleo de las FAS habrá que hacerlo con una Doctrina Conjunta.

Para terminar, solo cabe añadir que la fuerza aérea de nuestro Ejército del Aire posee unas capacidades muy adecuadas para:

- Reaccionar con gran rapidez ante una crisis.

- Desplegar o intervenir antes que ninguna otra fuerza, incluso a grandes distancias.

- Proporcionar con medios aéreos o espaciales vigilancia y reconocimiento sin vulnerar soberanías.

- Controlar el espacio aéreo y proteger el despliegue de otras fuerzas con una indispensable sombrilla protectora.

- Adaptarse con gran flexibilidad a la escalada/desescalada del conflicto.

- Llegado el caso, concentrar su gran poder de fuego o atacar objetivos puntuales con gran precisión y sin daños colaterales.

- Gran visibilidad con el consiguiente efecto demostrativo o disuasorio.

- No tiene el riesgo de convertir a nuestros soldados de pacificadores en rehenes, facilitando una retirada rápida y oportuna del conflicto.

Por todo ello, los medios aéreos suponen un instrumento extremadamente útil para que en cualquier caso y, como siempre ha de ocurrir en el empleo del poder militar, la aplicación de la Fuerza Aérea ha de hacerse con un ajuste muy preciso a los objetivos políticos que se quieren alcanzar, con estricta sujeción a las limitaciones que se establezcan y contemplando todas las alternativas posibles de evolución de las crisis para ser capaces de escalar y desescalar cuando proceda. 